\title{
Intrinsic charm content of the nucleon and charmness-nucleon sigma term
}

\author{
Shaorong Duan ${ }^{1}$, C. S. An ${ }^{1} * *$ and B. Saghai2 \\ 1. School of Physical Science and Technology, \\ Southwest University, Chongqing 400715, People's Republic of China \\ 2. Université Paris-Saclay, Institut de Recherche sur les lois Fondamentales de l'Univers, \\ Irfu-CEA, F-91191 Gif-sur-Yvette, France
}

(Dated: June 8, 2016)

\begin{abstract}
In the extended chiral constituent quark model, the intrinsic $c \bar{c}$ content of the nucleon is investigated. The probabilities of the quark-antiquark components in the nucleon wave functions are calculated by taking the nucleon to be admixtures of three- and five-quark components, with the relevant transitions handled via the ${ }^{3} \mathrm{P}_{0}$ mechanism. Predictions for the probability of the $c \bar{c}$ in the nucleon wave function and the charmness-nucleon sigma term are presented. Our numerical results turn out to be consistent with the predictions from various other approaches reported in the literature.
\end{abstract}

PACS numbers: 12.39.-x, 12.38.Lg, 14.65.Bt, 14.65.Dw

*Electronic address: ancs@swu.edu.cn

${ }^{\dagger}$ Electronic address: bijan.saghai@cea.fr 


\section{INTRODUCTION}

Intrinsic quark-antiquark content of the nucleon is a prediction of Quantum Chromodynamics (QCD). Recent review papers witness of the ongoing extensive theoretical and experimental efforts since about four decades on the light and heavy quark-antiquark pairs in the baryons; see e.g. [1-3]].

In 1980s, Brodsky and collaborators [4, 5] postulated the existence of the $|u u d c \bar{c}\rangle$ components in the proton in order to account for the large cross section of charmness production in the proton-proton collisions. They coined the term "intrinsic" to be distinguished from the extrinsic contributions arising from gluon splitting in perturbative QCD. The outcome of the developed light-cone formalism is known as the BHPS model and suggests a probability of $\mathcal{P}_{N}^{c \bar{c}} \approx 1 \%$ for the intrinsic $c \bar{c}$ (IC) component in the proton. Following that pioneer work, various phenomenological approaches were developed to extract $\mathcal{P}_{N}^{c \bar{c}}$ from data, such as photon-gluon fusion [6 8], meson cloud model [9] and global QCD analysis of parton distribution [10], leading to $\mathcal{P}_{N}^{c \bar{c}} \approx 0.3-1(\%)$. Pumplin and collaborators [11] removed some simplification assumptions of the BHPS model and evaluated the sensitivity of the hardscattering data to the IC, concluding that the corresponding probability can range between zero and 3\%. More recently Dulat and collaborators [12] analyzed the parton distribution function $(\mathrm{PDF})$ of the proton based on the NNLO approximation of perturbative QCD and included the combined H1 and ZEUS data [13], reaching the conclusion that the PDF uncertainties are just as large as the IC effects. In summary, the nucleon's IC remains elusive, however several studies predict measurable effects of such possible components in the ongoing and/ or forthcoming experiments at the LHC [12, 14 20] and RHIC [15, 21, 22].

Another important entity in this realm is the charmness-nucleon sigma term $\sigma_{c N}$, related to the explicit breaking of chiral symmetry. In recent years, few LQCD results became available by the ETM [23] and $\chi$ QCD [24] Collaborations. It can also be extracted from another LQCD calculation performed by the MILC Collaboration [25]. The central values coming from those works lie in the range of $67-94 \mathrm{MeV}$, albeit with large uncertainties $\approx(30-50) \%$, making all results consistent with each other.

Phenomenologically, genuine higher Fock states in the baryons' wave functions constitute a pertinent nonperturbative source of the intrinsic $Q \bar{Q}$ components. In our recent works [26, 27] we studied those components in baryons, with $Q \equiv u, d, s$, and the associated sigma 
terms. In the present work we extend our approach to the intrinsic $c \bar{c}$ content of the nucleon and the charmness-nucleon sigma term. Here we derive the wave functions for all possible quark-antiquark components in the nucleon, and calculate the corresponding probability amplitudes using the ${ }^{3} P_{0}$ quark-antiquark creation model [28]. Also the resulting charmnessnucleon sigma term is evaluated.

The present manuscript is organized in the following way: in sec. [II after a brief presentation of the theoretical frame, we give explicit expressions for the sigma terms relating them to the quark-antiquark pair probabilities. Numerical results for the probabilities of light, strange and charm quark-antiquark pairs in the nucleon, as well as $\sigma_{\pi N}, \sigma_{s N}$ and $\sigma_{c N}$ are reported in sec. III and compared to findings from other sources. Finally, sec. IV contains a summary and conclusions.

\section{THEORETICAL FRAME}

The extended chiral constituent quark model on the light and strangeness components of baryons was developed in [26], and applied to the sigma terms of baryons in [27]. So, here we briefly present the main content of the formalism and extend it to the charm sector.

\section{A. The extended chiral constituent quark model}

In the extended chiral constituent quark model, wave function for the nucleon reads,

$$
|\psi\rangle_{N}=\frac{1}{\sqrt{\mathcal{N}}}\left[|q q q\rangle+\sum_{i, n_{r}, l} C_{i n_{r} l}\left|q q q(Q \bar{Q}), i, n_{r}, l\right\rangle\right]
$$

where the first term is the conventional wave function for the nucleon with three constituent quarks $(q \equiv u, d)$ and the second term is a sum over all possible higher Fock components with a $Q \bar{Q}$ pair; $Q \bar{Q} \equiv u \bar{u}, d \bar{d}, s \bar{s}, c \bar{c}$. Different possible orbital-flavor-spin-color configurations of the four-quark subsystems in the five-quark system, numbered by $i ; n_{r}$ and $l$, denote the inner radial and orbital quantum numbers, respectively, while $C_{i n_{r} l} / \sqrt{\mathcal{N}}$ represents the probability amplitude for the corresponding five-quark component. As discussed explicitly in [26], here we only need to consider the five-quark configurations with $n_{r}=0$ and $l=1$, consequently, there are 17 different configurations which can be classified in four categories according to the orbital and spin wave functions of the four-quark subsystem; the 
TABLE I: Categories ( $2^{\text {nd }}$ line) and configurations (lines 3-8) for five-quark components.

\begin{tabular}{|c|c|c|c|c|c|c|}
\hline $\begin{array}{c}\text { i Category / Config. } \\
\text { I / }[31]_{X}[22]_{S}\end{array}$ & $\mathrm{i}$ & $\begin{array}{l}\text { Category / Config. } \\
\qquad \text { II / }[31]_{X}[31]_{S}\end{array}$ & $\mathrm{i}$ & $\begin{array}{l}\text { Category / Config. } \\
\text { III / }[4]_{X}[22]_{S}\end{array}$ & $\mathrm{i}$ & $\begin{array}{c}\text { Category / Config. } \\
\qquad \mathrm{IV} /[4]_{X}[31]_{S}\end{array}$ \\
\hline $1[31]_{X}[4]_{F S}[22]_{F}[22]_{S}$ & 5 & {$[31]_{X}[4]_{F S}[31]_{F}^{1}[31]_{S}$} & 11 & {$[4]_{X}[31]_{F S}[211]_{F}[22]_{S}$} & & {$[4]_{X}[31]_{F S}[211]_{F}[31]_{S}$} \\
\hline $2[31]_{X}[31]_{F S}[211]_{F}[22]_{S}$ & 6 & {$[31]_{X}[4]_{F S}[31]_{F}^{2}[31]_{S}$} & 12 & {$[4]_{X}[31]_{F S}[31]_{F}^{1}[22]_{S}$} & & {$[4]_{X}[31]_{F S}[22]_{F}[31]_{S}$} \\
\hline $3[31]_{X}[31]_{F S}[31]_{F}^{1}[22]_{S}$ & 7 & {$[31]_{X}[31]_{F S}[211]_{F}[31]_{S}$} & 13 & {$[4]_{X}[31]_{F S}[31]_{F}^{2}[22]_{S}$} & 16 & {$[4]_{X}[31]_{F S}[31]_{F}^{1}[31]_{S}$} \\
\hline \multirow[t]{3}{*}{$4[31]_{X}[31]_{F S}[31]_{F}^{2}[22]_{S}$} & 8 & {$[31]_{X}[31]_{F S}[22]_{F}[31]_{S}$} & & & 17 & {$[4]_{X}[31]_{F S}[31]_{F}^{2}[31]_{S}$} \\
\hline & 9 & {$[31]_{X}[31]_{F S}[31]_{F}^{1}[31]_{S}$} & & & & \\
\hline & 10 & {$[31]_{X}[31]_{F S}[31]_{F}^{2}[31]_{S}$} & & & & \\
\hline
\end{tabular}

corresponding configurations are listed in Table [, using the shorthand notation for Young tableaux. Note that, the charmness configurations with flavor symmetry $[31]_{F}^{1}$ cannot form Fock components of the nucleon.

In Table II, we construct the explicit wave functions of the studied flavor configurations of the four-quark subsystem in the charmness components in the nucleon. To estimate the energies of the studied hidden charm pentaquark configurations, we employ the chiral constituent quark model developed in [26]. As discussed in that reference, all the configurations share a same energy $E_{0}=2127 \mathrm{MeV}$, if the difference between the constituent masses of

TABLE II: Flavor wave functions of the charmness configurations studied here. Note that, the full wave functions are obtained by multiplying each column by the corresponding normalization factor.

\begin{tabular}{|c|c|c|c|c|c|c|c|c|}
\hline & {$[22]_{F_{1}}$} & {$[22]_{F_{2}}$} & {$[31]_{F_{1}}^{2}$} & {$[31]_{F_{2}}^{2}$} & {$[31]_{F_{3}}^{2}$} & {$[211]_{F_{1}}$} & {$[211]_{F_{2}}$} & {$[211]_{F_{3}}$} \\
\hline$u u d c$ & 2 & 0 & 0 & 6 & 0 & 2 & 0 & 0 \\
\hline uucd & 2 & 0 & 2 & 2 & 0 & -2 & 0 & 0 \\
\hline dcuu & 2 & 0 & -1 & -4 & -2 & 0 & 2 & 1 \\
\hline cduu & 2 & 0 & -1 & -4 & 2 & 0 & -2 & -1 \\
\hline duuc & -1 & -1 & 0 & -3 & -3 & -1 & -3 & 0 \\
\hline$u d u c$ & -1 & 1 & 0 & -3 & 3 & -1 & 3 & 0 \\
\hline$c u d u$ & -1 & 1 & -1 & 5 & -1 & -1 & -1 & 1 \\
\hline$u c d u$ & -1 & -1 & -1 & 5 & 1 & -1 & 1 & -1 \\
\hline cuud & -1 & -1 & 2 & -1 & -1 & 1 & 3 & 0 \\
\hline$d u c u$ & -1 & 1 & -1 & -1 & -3 & 1 & 1 & -1 \\
\hline ucud & -1 & 1 & 2 & -1 & 1 & 1 & -3 & 0 \\
\hline$u d c u$ & -1 & -1 & -1 & -1 & 3 & 1 & -1 & 1 \\
\hline
\end{tabular}


charm and light quarks and the hyperfine interaction between quarks are not taken into account. Consequently, the energy $E_{i}$ for the $i^{\text {th }}$ configuration reads,

$$
E_{i}=E_{0}+2 \delta m+E_{i}^{h}
$$

where $\delta m=m_{c}-m_{q}$ is the constituent mass difference between charm and light quarks, and $E_{i}^{h}$ the energy caused by hyperfine interaction between quarks. To consider the hyperfine interaction between quarks, we employ the flavor-spin dependent version in the chiral constituent quark model [29],

$$
\begin{aligned}
H_{h}= & -\sum_{i<j} \vec{\sigma}_{i} \cdot \vec{\sigma}_{j}\left[\sum_{a=1}^{3} V_{\pi}\left(\vec{r}_{i j}\right) \lambda_{i}^{a} \lambda_{j}^{a}+\sum_{a=4}^{7} V_{K}\left(\vec{r}_{i j}\right) \lambda_{i}^{a} \lambda_{j}^{a}+V_{\eta}\left(\vec{r}_{i j}\right) \lambda_{i}^{8} \lambda_{j}^{8}\right. \\
& \left.+\sum_{a=9}^{12} V_{D}\left(\vec{r}_{i j}\right) \lambda_{i}^{a} \lambda_{j}^{a}+\sum_{a=13}^{14} V_{D_{s}}\left(\vec{r}_{i j}\right) \lambda_{i}^{a} \lambda_{j}^{a}+V_{\eta_{c}}\left(\vec{r}_{i j}\right) \lambda_{i}^{15} \lambda_{j}^{15}\right],
\end{aligned}
$$

where $\lambda_{i}^{a}$ denotes the $S U(4)$ Gell-Mann matrix acting on the $i^{t h}$ quark, $V_{M}\left(r_{i j}\right)$ is the potential of the $M$ meson-exchange interaction between $i^{\text {th }}$ and $j^{\text {th }}$ quark, as extensively discussed in [29, 30]. Then, $E_{i}^{h}$ is obtained by

$$
\begin{aligned}
E_{i}^{h}= & \left\langle Q Q Q(Q \bar{Q}), i, 0,1\left|H_{h}\right| Q Q Q(Q \bar{Q}), i, 0,1\right\rangle \\
= & -6 \sum_{n j k l m}\left[\left(C_{[31]_{i}^{n}[211]_{n}}^{\left[1^{14}\right]}\right)_{\left.\left[{ }_{[\mathcal{F}}\right]_{i}^{j} \mid \mathcal{X}\right]_{i}}^{[31]^{n}} C_{[\mathcal{F}]_{i}^{k}[\mathcal{X}]_{i}^{m}}^{\left[31^{n}\right.}\right. \\
& \left(\left\langle[\mathcal{X}]_{i}^{l}\left|V_{\pi}\left(r_{12}\right)\right|[\mathcal{X}]_{i}^{m}\right\rangle\left\langle[\mathcal{F} \mathcal{S}]_{i}^{j}\left|\vec{\sigma}_{1} \cdot \vec{\sigma}_{2} \sum_{a=1}^{3} \lambda_{1}^{a} \lambda_{2}^{a}\right|[\mathcal{F} \mathcal{S}]_{i}^{k}\right\rangle\right. \\
& +\left\langle[\mathcal{X}]_{i}^{l}\left|V_{K}\left(\vec{r}_{12}\right)\right|[\mathcal{X}]_{i}^{m}\right\rangle\left\langle[\mathcal{F} \mathcal{S}]_{i}^{j}\left|\vec{\sigma}_{1} \cdot \vec{\sigma}_{2} \sum_{a=4}^{7} \lambda_{1}^{a} \lambda_{2}^{a}\right|[\mathcal{F} \mathcal{S}]_{i}^{k}\right\rangle \\
& +\left\langle[\mathcal{X}]_{i}^{l}\left|V_{\eta}\left(\vec{r}_{12}\right)\right|[\mathcal{X}]_{i}^{m}\right\rangle\left\langle[\mathcal{F} \mathcal{S}]_{i}^{j}\left|\vec{\sigma}_{1} \cdot \vec{\sigma}_{2} \lambda_{1}^{8} \lambda_{2}^{8}\right|[\mathcal{F} \mathcal{S}]_{i}^{k}\right\rangle \\
& +\left\langle[\mathcal{X}]_{i}^{l}\left|V_{D}\left(\vec{r}_{12}\right)\right|[\mathcal{X}]_{i}^{m}\right\rangle\left\langle[\mathcal{F} \mathcal{S}]_{i}^{j}\left|\vec{\sigma}_{1} \cdot \vec{\sigma}_{2} \sum_{a=9}^{12} \lambda_{1}^{a} \lambda_{2}^{a}\right|[\mathcal{F} \mathcal{S}]_{i}^{k}\right\rangle \\
& +\left\langle[\mathcal{X}]_{i}^{l}\left|V_{D_{s}}\left(\vec{r}_{12}\right)\right|[\mathcal{X}]_{i}^{m}\right\rangle\left\langle[\mathcal{F} \mathcal{S}]_{i}^{j}\left|\vec{\sigma}_{1} \cdot \vec{\sigma}_{2} \sum_{a=13}^{14} \lambda_{1}^{a} \lambda_{2}^{a}\right|[\mathcal{F} \mathcal{S}]_{i}^{k}\right\rangle \\
& \left.\left.+\left\langle[\mathcal{X}]_{i}^{l}\left|V_{\eta_{c}}\left(\vec{r}_{12}\right)\right|[\mathcal{X}]_{i}^{m}\right\rangle\left\langle[\mathcal{F} \mathcal{S}]_{i}^{j}\left|\vec{\sigma}_{1} \cdot \vec{\sigma}_{2} \lambda_{1}^{15} \lambda_{2}^{15}\right|[\mathcal{F} \mathcal{S}]_{i}^{k}\right\rangle\right)\right],
\end{aligned}
$$

where $[\mathcal{F S}]_{i}^{N}$ and $[\mathcal{X}]_{i}^{N}$ represent the $N^{\text {th }}$ flavor-spin and orbital wave functions of the four-quark subsystem in the five-quark configuration with number $i$ of the 17 five-quark configurations. $C_{[31]_{i}^{n}[211]_{n}}^{\left[1^{4}\right]}, C_{[\mathcal{F S}]_{i}^{j}[\mathcal{X}]_{i}^{l}}^{[33]^{n}}$ and $C_{\left[\left.\mathcal{F} \mathcal{S}\right|_{i} ^{n}[\mathcal{X}]_{i}^{m}\right.}^{\left[31 n^{n}\right.}$ are the $S_{4}$ Clebsch-Gordan coefficients.

On the other hand, we have to consider the $S U(4)$ flavor symmetry breaking effects because of the large difference between the light and charm quark constituent masses. As 
introduced in [31], these effects can be calculated by using the following flavor-dependent Hamiltonian

$$
H_{s b}=-\sum_{i=1}^{4} \frac{m_{c}-m_{q}}{2 m_{q}}\left\{\frac{p_{i}^{2}}{m_{c}}+\frac{p_{\bar{c}}^{2}}{m_{c}}\right\} \delta_{i c}
$$

where $\delta_{i c}$ is a flavor dependent operator with eigenvalue 1 for charm quark and 0 for light quark. Here $m_{q}$ is the constituent mass of the light quark.

The coefficient $C_{i n_{r} l}$ for a given five-quark component can be related to the transition matrix element between the three- and five-quark configurations of the studied baryon. To calculate the corresponding transition matrix element, we use a ${ }^{3} \mathrm{P}_{0}$ version for the transition coupling operator $\hat{T}$,

$$
\begin{aligned}
\hat{T}= & -\gamma \sum_{j} \mathcal{F}_{j, 5}^{00} \mathcal{C}_{j, 5}^{00} C_{O F S C} \sum_{m}\langle 1, m ; 1,-m \mid 00\rangle \chi_{j, 5}^{1, m} \\
& \mathcal{Y}_{j, 5}^{1,-m}\left(\vec{p}_{j}-\vec{p}_{5}\right) b^{\dagger}\left(\vec{p}_{j}\right) d^{\dagger}\left(\vec{p}_{5}\right),
\end{aligned}
$$

with $\gamma$ a dimensionless constant of the model, $\mathcal{F}_{i, 5}^{00}$ and $\mathcal{C}_{i, 5}^{00}$ the flavor and color singlet of the quark-antiquark pair $Q_{i} \bar{Q}$ in the five-quark system, and $C_{O F S C}$ an operator to calculate the orbital-flavor-spin-color overlap between the residual three-quark configuration in the five-quark system and the valence three-quark system.

The probability of the quark-antiquark pairs in the nucleon and the normalization factor read, respectively,

$$
\begin{aligned}
\mathcal{P}^{Q \bar{Q}} & =\frac{1}{\mathcal{N}} \sum_{i=1}^{17}\left[\left(\frac{T_{i}^{Q \bar{Q}}}{M_{N}-E_{i}^{Q \bar{Q}}}\right)^{2}\right] \\
\mathcal{N} & \equiv 1+\sum_{i=1}^{17} \mathcal{N}_{i}=1+\sum_{i=1}^{17} \sum_{Q \bar{Q}}\left[\left(\frac{T_{i}^{Q \bar{Q}}}{M_{B}-E_{i}^{Q \bar{Q}}}\right)^{2}\right] .
\end{aligned}
$$

where the first term in Eq. (8) is due to the valence three-quark state, while the second term comes from the five-quark mixtures.

To derive the explicit wave functions of the five-quark components with light quarkantiquark pairs in the nucleon, we construct the wave functions for $u \bar{u}$ and $d \bar{d}$ components of the 17 different flavor-spin configurations to form the isospin state $\left|\frac{1}{2}, \frac{1}{2}\right\rangle$. The five-quark components with $s \bar{s}$ and $c \bar{c}$ pairs form the nucleon isospin naturally since those pairs do not contribute to isospin. Accordingly, starting from Eq. (8), the light quark-antiquark pairs $(u \bar{u}$ and $d \bar{d})$ probabilities for the nucleon in terms of the five-quark probabilities per configuration $\left(P_{N}(i), i=1-17\right)$ are combined [27] with the relevant squared Clebsch-Gordan 
coefficients in $S U(2)$ isospin space. For the $s \bar{s}$ and $c \bar{c}$ components, the probabilities $\mathcal{P}_{N}^{s \bar{s}}$ and $\mathcal{P}_{N}^{c \bar{c}}$ are obtained by summing up linearly the relevant nonvanishing contributions, $P_{N}^{s \bar{s}}(i)$ and $P_{N}^{c \bar{c}}(i)(\mathrm{i}=1,17)$, respectively.

\section{B. Sigma terms}

Here we proceed in line with Ref. [27], where explicit expressions for the pion- and strangeness-baryon sigma terms were given as a function of quark-antiquark pairs probabilities. Accordingly, the charmness-nucleon $\sigma$ term is defined as follows:

$$
\sigma_{c_{N}}=m_{c}\langle N|c \bar{c}| N\rangle
$$

which can be related to $\sigma_{\pi N}$

$$
\begin{aligned}
\sigma_{c N} & =\frac{m_{c}}{m_{l}} \frac{\langle N|c \bar{c}| N\rangle}{\langle N|u \bar{u}+d \bar{d}| N\rangle} \sigma_{\pi N} \\
& =\frac{m_{c}}{m_{l}} \frac{2 \mathcal{P}_{N}^{c \bar{c}}}{3+2\left(\mathcal{P}_{N}^{u \bar{u}}+\mathcal{P}_{N}^{d \bar{d}}\right)} \sigma_{\pi N}
\end{aligned}
$$

where

$$
\begin{aligned}
\sigma_{\pi N} & =\frac{\hat{\sigma}}{1-2(\langle N|s \bar{s}| N\rangle /\langle N|u \bar{u}+d \bar{d}| N\rangle)} \\
& =\frac{3+2\left(\mathcal{P}_{N}^{u \bar{u}}+\mathcal{P}_{N}^{d \bar{d}}\right)}{3+2\left(\mathcal{P}_{N}^{u \bar{u}}+\mathcal{P}_{N}^{d \bar{d}}-2 \mathcal{P}_{N}^{s \bar{s}}\right)} \hat{\sigma}
\end{aligned}
$$

with $m_{l} \equiv\left(m_{u}+m_{d}\right) / 2$ the average current mass of the up and down quarks; $m_{s}$ and $m_{c}$ the current mass of the strange and charm quarks, respectively, and $\hat{\sigma}$ the nucleon expectation value of the purely octet operator.

\section{RESULTS AND DISCUSSION}

First we present the model parameters. As documented in [32], the input parameters of the model for $u \bar{u}, d \bar{d}$ and $s \bar{s}$ are taken from the literature. The newly introduced ones concern the charm quark, namely, its mass, and the hyperfine interaction strength between the light and charm quarks. For the former one, we use the empirical value $m_{c}=1275(25) \mathrm{MeV}$ given in PDG [33], and the latter one is taken from Ref. [30]. 
The only source of uncertainty in the probabilities, presented in sec. IIIA, comes from a common factor of the matrix elements of the transitions between three- and five-quark components and was found [26] to be $V=570 \pm 46 \mathrm{MeV}$, by successfully fitting the experimental data for the proton flavor asymmetry $\bar{d}-\bar{u} \equiv \mathcal{P}_{p}^{d \bar{d}}-\mathcal{P}_{p}^{u \bar{u}}=0.118 \pm 0.012$ [34]. Introduction of the five-quark components with the charm quark-antiquark pairs and fitting the same data point, the new extracted value is $V=572 \pm 47 \mathrm{MeV}$, differing by $0.4 \%$ from the previous one. For the $\sigma$-terms two additional entities contribute to the uncertainties [27], namely, the nonsinglet component $\hat{\sigma}=33(5) \mathrm{MeV}$, as extracted within the chiral perturbation theory [35] and the PDG masses ratio [33] $m_{s} / m_{l}=27.5(1.0)$. Accordingly, compared to our previous studies [26, 27] only one parameter was slightly readjusted in the frame of the present work.

In this section, we report our numerical results for the probabilities of the quark-antiquark components in the nucleon and the relevant sigma terms, followed by comparisons to findings by other authors.

\section{A. Numerical results}

Table III embodies our numerical results. In columns 3 to 5 the quark-antiquark pairs probabilities in the nucleon per configuration are given for light, strange and charm components, respectively. The total five quark-antiquark probabilities are reported in column 6 . The pion-, strangeness- and charmness-sigma terms are given in columns 7 to 9 , respectively.

Note that,the numerical results for the light and strange quark-antiquark pairs (columns 3-4 and 7-8), reported in [27] for $V=570 \pm 46 \mathrm{MeV}$, are given here with the updated value for $V$ and allow us to make clear the relative weight of the $c \bar{c}$ component and the discussion on the sigma terms at the end of this section.

As reported in Table III, out of the 17 five-quark configurations in the nucleon, only 3 of them contribute to all the light, $s \bar{s}$ and $c \bar{c}$ pairs probabilities, whereas 5 of them have only $u \bar{u}$ and / or $d \bar{d}$ components, while the remaining 9 configurations are exclusively composed of $s \bar{s}$ and $c \bar{c}$ pairs.

In the light quark-antiquark sector, the five-quark probability is dominated by the first category, where the total spin of the four-quark subsystem is 0 . Within that category, the configuration $n^{\circ} 1$ gives the largest contribution and corresponds to the configuration with the lowest energy and largest coupling to the three-quark component. 
TABLE III: Predictions for probabilities of different five-quark configurations for the nucleon (in \%), with $\mathcal{P}_{N}^{q \bar{q}}=\mathcal{P}_{N}^{u \bar{u}}+\mathcal{P}_{N}^{d \bar{d}}, \mathcal{P}_{N}^{Q \bar{Q}}=\mathcal{P}_{N}^{q \bar{q}}+\mathcal{P}_{N}^{s \bar{s}}+\mathcal{P}_{N}^{c \bar{c}}$, and pion-, strangeness- and charmness-nucleon sigma terms (in $\mathrm{MeV})$.

\begin{tabular}{|c|c|c|c|c|c|c|c|c|}
\hline $\mathrm{i}$ & Category & $\mathcal{P}_{N}^{q \bar{q}}$ & $\mathcal{P}_{N}^{s \bar{s}}$ & $\mathcal{P}_{N}^{c \bar{c}}$ & $\mathcal{P}_{N}^{Q \bar{Q}}$ & $\sigma_{\pi N}$ & $\sigma_{s N}$ & $\sigma_{c N}$ \\
\hline & I) $[31]_{X}[22]_{S}$ : & & & & & & & \\
\hline 1 & & $14.58(1.50)$ & $0.98(10)$ & $0.04(0)$ & $15.60(1.60)$ & $33.4(5.1)$ & $5.5(1.5)$ & $3.0(8)$ \\
\hline 2 & & 0 & $0.36(4)$ & $0.03(1)$ & $0.39(4)$ & $33.2(5.0)$ & $2.2(6)$ & $2.7(7)$ \\
\hline 3 & & $1.64(17)$ & 0 & 0 & $1.64(17)$ & $33.0(5.0)$ & 0 & 0 \\
\hline \multirow[t]{3}{*}{4} & & 0 & $0.26(3)$ & $0.03(1)$ & $0.29(3)$ & $33.1(5.0)$ & $1.6(5)$ & $2.6(7)$ \\
\hline & Category I & $16.22(1.66)$ & $1.60(16)$ & $0.10(1)$ & $17.92(1.83)$ & $33.6(5.2)$ & $8.9(2.5)$ & $7.8(2.1)$ \\
\hline & II) $[31]_{X}[31]_{S}$ : & & & & & & & \\
\hline 5 & & $7.27(75)$ & 0 & 0 & $7.27(75)$ & $33.0(5.0)$ & 0 & 0 \\
\hline 6 & & 0 & $0.63(6)$ & $0.04(0)$ & $0.67(6)$ & $33.3(5.1)$ & $3.8(1.1)$ & $3.2(9)$ \\
\hline 7 & & 0 & $0.32(4)$ & $0.03(1)$ & $0.36(3)$ & $33.1(5.0)$ & $2.0(6)$ & $2.6(7)$ \\
\hline 8 & & $0.61(6)$ & $0.18(2)$ & $0.02(0)$ & $0.81(8)$ & $33.1(5.0)$ & $1.1(3)$ & $1.5(4)$ \\
\hline 9 & & $0.47(5)$ & 0 & 0 & $0.47(5)$ & $33.0(5.0)$ & 0 & 0 \\
\hline \multirow[t]{3}{*}{10} & & 0 & $0.08(1)$ & $0.01(0)$ & $0.09(1)$ & $33.0(5.0)$ & $0.5(1)$ & $0.8(2)$ \\
\hline & Category II & $8.33(0.86)$ & $1.21(13)$ & $0.10(1)$ & $9.64(98)$ & $33.5(5.1)$ & $7.0(2.0)$ & $7.8(2.1)$ \\
\hline & III) $[4]_{X}[22]_{S}:$ & & & & & & & \\
\hline 11 & & 0 & $0.85(9)$ & $0.09(1)$ & $0.94(10)$ & $33.4(5.1)$ & $5.2(1.5)$ & $7.2(2.0)$ \\
\hline 12 & & $4.13(42)$ & 0 & 0 & $4.13(42)$ & $33.0(5.0)$ & 0 & 0 \\
\hline \multirow[t]{3}{*}{13} & & 0 & $0.65(7)$ & $0.09(1)$ & $0.74(8)$ & $33.3(5.1)$ & $4.0(1.2)$ & $7.0(1.9)$ \\
\hline & Category III & $4.13(42)$ & $1.50(16)$ & $0.18(2)$ & $5.81(60)$ & $33.7(5.2)$ & $9.0(2.6)$ & $14.0(3.8)$ \\
\hline & IV) $[4]_{X}[31]_{S}:$ & & & & & & & \\
\hline 14 & & 0 & $0.77(8)$ & $0.09(1)$ & $0.86(9)$ & $33.3(5.1)$ & $4.7(1.4)$ & $7.1(2.0)$ \\
\hline 15 & & $1.49(16)$ & $0.44(5)$ & $0.06(1)$ & $1.99(21)$ & $33.2(5.0)$ & $2.6(8)$ & $4.5(1.2)$ \\
\hline 16 & & $1.18(12)$ & 0 & 0 & $1.18(12)$ & $33.0(5.0)$ & 0 & 0 \\
\hline \multirow[t]{3}{*}{17} & & 0 & $0.19(2)$ & $0.03(1)$ & $0.22(2)$ & $33.1(5.1)$ & $1.1(3)$ & $2.2(6)$ \\
\hline & Category IV & $2.67(28)$ & $1.40(15)$ & $0.18(2)$ & $4.25(44)$ & $33.6(5.2)$ & $8.5(2.5)$ & $13.8(3.8)$ \\
\hline & ll configurations & $31.35(3.21)$ & $5.71(59)$ & $0.56(6)$ & $37.62(3.85)$ & $35.2(5.5)$ & $30.5(8.5)$ & $39.3(10.3)$ \\
\hline
\end{tabular}

In the case of $\mathcal{P}_{N}^{s \bar{s}}$, the four categories have comparable contributions, though the first one gives the highest probability; where the total spin of the four-quark subsystem is $S=1$ and the total angular momentum is $J=0$.

Finally, for $\mathcal{P}_{N}^{c \bar{c}}$, the last two categories contribute almost equally, but with larger probabilities than the first two ones. While the category III corresponds to the four-quark 
subsystem $J=0$, in the category IV the total spin of the four-quark subsystem should be $S_{[31]}=1$ and $J=S_{4} \oplus L_{\bar{q}}=0$.

With respect to the sigma terms, the pion-nucleon $\sigma$ term of every configuration is $\approx 33.6 \mathrm{MeV}$, very close to the complete calculation with all 17 configurations leading to $\approx 35 \mathrm{MeV}$. But the strangeness- and charmness-nucleon sigma terms per configuration are about a factor of 4 to more than one order of magnitude smaller than the total of all configurations. Accordingly, any configuration truncated model will significantly underestimate both $\sigma_{s N}$ and $\sigma_{c N}$, leading to confusing results.

\section{B. Discussion and comparisons to previous results}

Probabilities and sigma terms related to the light and strange quark-antiquark sector were presented and discussed in our previous study [27]. Therein, the determined probabilities came out compatible with those reported within the generalized BHPS approach [36] and the meson cloud model [37]. Also the sigma terms related to the light and strange quarkantiquark sector turned out to be in good agreement with results coming from various approaches, namely, chiral Lagrangian [38], chiral perturbation theory [39], and LQCD [40]. In this section we hence concentrate on the charm issues.

\section{Quark-antiquark probabilities in the nucleon}

In Table IV probabilities for the $c \bar{c}$ pairs in the nucleon are reported.

TABLE IV: Predictions for the probability of $c \bar{c}$ in the nucleon (\%).

\begin{tabular}{|c|c|c|}
\hline Reference & Approach & $\mathcal{P}_{N}^{c \bar{c}}$ \\
\hline Present work & $\mathrm{E} \chi \mathrm{CQM}$ & $0.6(1)$ \\
\hline Brodsky et al. [4] & Light-cone & $\approx 1$ \\
\hline Hoffmann and Moore, [6] & PGF - NLO & 0.31 \\
\hline Harris et al. [7] & PGF - NLO & $0.86(60)$ \\
\hline Martin et al. [8] & NNLO & 0.3 \\
\hline Steffens et al. [9] & Meson cloud & $\approx 0.4$ \\
\hline Dulat et al. [12] & PQCD-NNLO & $\leq 2$ \\
\hline Jimenez-Delgado [10] & $\mathrm{PDF}$ & $0.3-0.4 ; \approx 1$ \\
\hline
\end{tabular}

As mentioned in Introduction, the first calculations embodying intrinsic $c \bar{c}$ was performed 
by Brodsky and collaborators [4] within the light-cone Fock space framework, by introducing the hypothesis that $P_{N}^{c \bar{c}}$ could be around 1\%. Hoffmann and Moore, [6] investigated the matter within a photon-gluon fusion (PGF) model at NLO and also took into account the quark and target mass contributions to the charm cross section, finding a smaller probability: $P_{N}^{c \bar{c}}=0.31 \%$. Harris et al. [7] extended that work interpreting the EMC charm production data [41] by calculating next-to-leading order and generalizing it for both extrinsic and intrinsic contributions to the charm structure function and found an intrinsic charm probability of $(0.86 \pm 0.60) \%$. Steffens et al. [9] used a more extended data base for the charm structure function, including the very low-x region measurements by the H1 [42] and ZEUS [43] collaborations. The authors performed a consistent interpolation between the two asymptotic regions of massless evolution at large $Q^{2}$ and the PGF, finding a slight preference for $P_{N}^{c \bar{c}} \approx 0.4 \%$. Later Martin et al. [8], using very extensive data coming from some 40 data sets released between 1989 and 2008, updated the parton distribution functions determined from global analysis of hard-scattering data up to NNLO and found $P_{N}^{c \bar{c}}=0.3 \%$. In a recent work, Jimenez-Delgado et al. [10] report the results of a new global QCD analysis of parton distribution functions (PDF), concentrating on the momentum fraction carried by the intrinsic charm quarks in terms of the Feynman-x,

$$
\langle x\rangle_{c+\bar{c}}=\int_{0}^{1} x[c(x)+\bar{c}(x)] d x
$$

which is related to the $c \bar{c}$ content of the nucleon by,

$$
\mathcal{P}_{N}^{c \bar{c}}=\int_{0}^{1} c(x) d x=\int_{0}^{1} \bar{c}(x) d x
$$

Note that in the BHPS model the predictions [1] at the input scale $Q_{0}=m_{c}=1.3 \mathrm{GeV}$, are $\langle x\rangle_{c+\bar{c}}=0.57 \%$ and $\mathcal{P}_{N}^{c \bar{c}}=1 \%$. Jimenez-Delgado et al. [10] analyzed a large set of data with $\langle x\rangle_{c+\bar{c}}$ in the range of 0 to $\approx 0.6 \%$. Fitting only the EMC data, they obtained $\mathcal{P}_{N}^{c \bar{c}}$ $=0.3-0.4 \%$, while excluding the old EMC data led to $\langle x\rangle_{c+\bar{c}}=0.5 \%$; a value close to the BHPS prediction. The minimization approach in the former work raised a debate [44, 45] emphasizing the need for more precise data.

Then, a global conclusion on the probability of the intrinsic $c \bar{c}$ component in the nucleon is that its value would be in the range of 0.3 to $1 \%$ and our result, $0.6 \%$, falls in that range.

However, as briefly discussed below, the genuine $c \bar{c}$ component is predicted to play a significant role in the forthcoming measurements using high energy beams at CERN/LHC, BNL/RHIC... 
Actually, it is well established that the heavy quarks produced in line with perturbative QCD carry small longitudinal momentum, while the intrinsic heavy constituents transport the largest fraction of the momentum of the hadron. Accordingly, to probe the intrinsic charm in the nucleon various guidelines were elaborated.

Brodsky and collaborators [14] proposed a fixed target experiment for the LHC $7 \mathrm{TeV}$ beam, allowing precise enough measurements of the rapidity distribution of open- or hiddencharm hadrons at $\sqrt{s_{N N}}=115 \mathrm{GeV}$, accessing the domain of high $x_{F}$; knowing that [4, 46] the intrinsic quark-antiquark possible manifestations should be looked for roughly in the range $0.2 \leq x_{F} \leq 0.8$.

Kniehl and collaborators [16] employed the general-mass variable-flavor-number scheme at NLO to study the inclusive production of the $D$ meson, $p p \rightarrow D^{\circ} X$, at the LHC and found that the production cross sections at $\sqrt{s_{N N}}=7 \mathrm{TeV}$ and large values of rapidity are sensitive to a non-perturbative component of the charm $\mathrm{PDF}$ for $\mathcal{P}_{N}^{c \bar{c}}=3.5 \%$.

Bednyakov and collaborators [17] reported results for $p p \rightarrow \gamma c X$ differential cross section at $\sqrt{s}=8 \mathrm{TeV}$. Calculations come from the radiatively generated charm PDF (CTEQ66), the sea-like PDF (CTEQ66c4) and the BHPS PDF (CTEQ66c2) also for $\mathcal{P}_{N}^{c \bar{c}}=3.5 \%$ and found that the IC manifestation could be measured with both the ATLAS and CMS detectors.

Bailas and Goncalves [18] studied, within various models, the impact of the IC on the rapidity and transverse momentum distribution in the Z-boson production in proton-proton collisions at the LHC, and showed that the $Z+c$ cross section is significantly sensitive to the presence of the IC.

Finally, the relevance of the CEBAF-12 GeV and FAIR-PANDA facilities to study the multiquark dynamics in baryons was also underlined [47].

\section{Sigma terms}

Compared to $\sigma_{\pi N}$ and, to a lesser extent to $\sigma_{s N}$, for the charmness-nucleon sigma term fewer results are available, coming from lattice QCD results as given in Table V. Note that, wherever appropriate, using statistical and systematic uncertainties reported in those papers, we give $\delta=\sqrt{\delta_{\text {stat }}^{2}+\delta_{\text {sys }}^{2}}$.

The most recent results were released by the ETM Collaboration [23], employing improved methods for the disconnected quark loops; determining $\sigma_{\pi N}, \sigma_{s N}$ and $\sigma_{c N}$. Comparing our 
results to those of the latter work, we note that the outcomes for $\sigma_{\pi N}$ and $\sigma_{s N}$ are in agreement within $1 \sigma$ (for a comprehensive discussion with extractions of these latter terms by other authors see [27] ). Our value for $\sigma_{c N}$ is compatible with the ETM result within $2 \sigma$. This is also the case for the strangeness content of the nucleon $y_{N}$, for which they get 0.075(16) compared to our approach's value 0.031(3). The same quantity for the charmless content of the nucleon within the present work is $y_{N}^{c}=0.004(1)$, but to our knowledge no other value was reported in the literature for that entity.

TABLE V: Predictions for the sigma terms $\sigma_{\pi N}, \sigma_{s N}$ and $\sigma_{c N}$ of the nucleon $(\mathrm{MeV})$.

\begin{tabular}{lcccc}
\hline \hline Reference (Collaboration) & Approach & $\sigma_{\pi N}$ & $\sigma_{s N}$ & $\sigma_{c N}$ \\
\hline Present work & E $\chi$ CQM & $35(6)$ & $30(8)$ & $39(10)$ \\
Abdel-Rehim et al. [23] (ETM) & LQCD & $37(7)$ & $41(8)$ & $79(22)$ \\
Gong et al. [24] ( $\chi$ QCD) & LQCD & & $33(6)$ & $94(31)$ \\
Freeman and Toussaint [25] (MILC) & LQCD & & $39(8)$ & $67(32)$ \\
\hline \hline
\end{tabular}

The $\chi$ QCD Collaboration [24] investigated the $Q \bar{Q}$ components within a dynamical LQCD with overlap valence quarks on $2+1$ flavors domain-wall fermion gauge configuration. They performed calculations for the strange and charm quark-antiquark contributions and determined both $\sigma_{s N}$ and $\sigma_{c N}$. For the strangeness sigma term the agreement between their result and ours is perfect, while for $\sigma_{c N}$ the two finding are compatible with each other within less than $2 \sigma$.

The MILC Collaboration [25] applied a hybrid method to the large library of improved staggered gauge configuration to calculate both matrix-elements $\langle N|s \bar{s}| N\rangle=0.44 \pm 0.08$ (stat) \pm 0.05 and $\langle N|c \bar{c}| N\rangle=0.058 \pm 0.027$ (stat). Using for the masses the values quoted by the authors, $m_{s}=89.0 \mathrm{MeV}$ and $m_{c}=1.2 \mathrm{GeV}$, we report in Table $\mathrm{V}$ the corresponding sigma terms. Here also we find good agreement with our results for $\sigma_{s N}$ and $\sigma_{c N}$ within $1 \sigma$.

\section{SUMMARY AND CONCLUSIONS}

Our recent works [26, 27] and the present study, performed within the extended chiral quark approach, constitute a thorough investigation of the genuine quark-antiquarks pairs in the nucleon. The quark-antiquark pairs creation was calculated via the ${ }^{3} P_{0}$ mechanism [28]. All possible five-quark configurations which may form higher Fock components of the nucleon 
were taken into account and it was shown that any configuration truncated calculation will lead to unrealistic results.

This coherent and comprehensive set of results allowed us to predict the probabilities of the $u \bar{u}, d \bar{d}, s \bar{s}$ and $c \bar{c}$ pairs in the nucleon as well as the associated sigma terms $\sigma_{\pi N}, \sigma_{s N}$ and $\sigma_{c N}$. The model uncertainties are about $10 \%$, mainly due to the only fitted parameter on the proton flavor asymmetry $\bar{d}-\bar{u}=0.118(12)$ [34]. All other parameters were taken from the literature [32].

Extensive comparisons with the outcomes of other approaches, reported in [26, 27] and the present paper led, in general, to compatibility of the obtained results with those found in the literature. To our knowledge, the present approach is the only available one putting forward predictions for all the above mentioned entities within a single approach and set of input parameters.

The predicted probabilities of the five-quark components with light, strange and charm quark-antiquark pairs in the nucleon wave function, turned out to be (in \%) $P_{N}^{q \bar{q}}=31.3(3.2$ ), $P_{N}^{s \bar{s}}=5.7(6)$ and $P_{N}^{c \bar{c}}=0.6(1)$, respectively, adding up to $P_{N}^{Q \bar{Q}}=37.6$ (3.8). As reported in [26] and Sec. IIIB 1, our findings are compatible with results released by several authors.

Here, three observations are in order: i) the intrinsic five-quark states represent a significant part of the nucleon wave function, ii) the probability of charm-anticharm pairs is rather tiny, iii) there is no non-ambiguous experimental evidence for the existence of heavy quarkantiquark pairs in the nonperturbative regime. However, as discussed in Sec. IIIB 1, the present state-of-the-art in experimental high-energy physics allows us anticipating crucial measurements at the LHC and RHIC [14-22]. Also empirical determination of the intrinsic charm through PDF analysis with heavy-quarks are foreseen to shed a valuable light on those issues; see e.g. [48, 49] and references therein.

In parallel, LQCD calculations are producing results for the charmless-nucleon sigma term [23 25]. Here also our determination of that entity is compatible with the LQCD findings. Refinements in the latter approach, expected to reduce the presently large uncertainties, will certainly offer a better understanding of the underlying mechanisms with respect to the role, if any, played by the charmness in the nucleon.

Those efforts will hopefully lead to uncovering the puzzle of possible charm components in the nucleon. 


\section{Acknowledgments}

We thank Y. Chen and L. S. Geng for the suggestion to consider the charmness-nucleon sigma term and useful discussions. This work is partly supported by the Chongqing Natural Science Foundation under Grant No. cstc2015jcyjA00032, and the Fundamental Research Funds for the Central Universities under Grants No. XDJK2015C150 and No. SWU115020.

[1] S. J. Brodsky, A. Kusina, F. Lyonnet, I. Schienbein, H. Spiesberger and R. Vogt, A review of the intrinsic heavy quark content of the nucleon, Adv. High Energy Phys. 2015, 231547 (2015).

[2] T. J. Hobbs, J. T. Londergan and W. Melnitchouk, Phenomenology of nonperturbative charm in the nucleon, Phys. Rev. D 89, 074008 (2014).

[3] W. C. Chang and J. C. Peng, Flavor Structure of the Nucleon Sea, Prog. Part. Nucl. Phys. 79, 95 (2014).

[4] S. J. Brodsky, P. Hoyer, C. Peterson and N. Sakai, The Intrinsic Charm of the Proton, Phys. Lett. B 93, 451 (1980).

[5] S. J. Brodsky, C. Peterson and N. Sakai, Intrinsic Heavy Quark States, Phys. Rev. D 23, 2745 (1981).

[6] E. Hoffmann and R. Moore, Subleading Contributions to the Intrinsic Charm of the Nucleon, Z. Phys. C 20, 71 (1983).

[7] B. W. Harris, J. Smith and R. Vogt, Reanalysis of the EMC charm production data with extrinsic and intrinsic charm at NLO, Nucl. Phys. B 461, 181 (1996).

[8] A. D. Martin, W. J. Stirling, R. S. Thorne and G. Watt, Parton distributions for the LHC, Eur. Phys. J. C 63, 189 (2009).

[9] F. M. Steffens, W. Melnitchouk and A. W. Thomas, Charm in the nucleon, Eur. Phys. J. C 11, 673 (1999).

[10] P. Jimenez-Delgado, T. J. Hobbs, J. T. Londergan and W. Melnitchouk, New limits on intrinsic charm in the nucleon from global analysis of parton distributions, Phys. Rev. Lett. 114, 082002 (2015).

[11] J. Pumplin, Light-cone models for intrinsic charm and bottom, Phys. Rev. D 73, 114015 
(2006). J. Pumplin, H. L. Lai and W. K. Tung, The Charm Parton Content of the Nucleon, Phys. Rev. D 75, 054029 (2007).

[12] S. Dulat et al., Intrinsic Charm Parton Distribution Functions from CTEQ-TEA Global Analysis, Phys. Rev. D 89, 073004 (2014).

[13] H. Abramowicz et al. [H1 and ZEUS Collaborations], Combination and QCD Analysis of Charm Production Cross Section Measurements in Deep-Inelastic ep Scattering at HERA, Eur. Phys. J. C 73, no. 2, 2311 (2013).

[14] S. J. Brodsky, F. Fleuret, C. Hadjidakis and J. P. Lansberg, Physics Opportunities of a FixedTarget Experiment using the LHC Beams, Phys. Rept. 522, 239 (2013).

[15] V. P. Goncalves and F. S. Navarra, Looking for intrinsic charm in the forward region at BNL RHIC and CERN LHC, Nucl. Phys. A 842, 59 (2010).

[16] B. A. Kniehl, G. Kramer, I. Schienbein and H. Spiesberger, Inclusive Charmed-Meson Production at the CERN LHC, Eur. Phys. J. C 72, 2082 (2012).

[17] V. A. Bednyakov et al., Searching for intrinsic charm in the proton at the LHC, Phys. Lett. B 728, 602 (2014).

[18] G. Bailas and V. P. Goncalves, Phenomenological implications of the intrinsic charm in the $Z$ boson production at the LHC, Eur. Phys. J. C 76, 105 (2016).

[19] R. D. Ball, V. Bertone, M. Bonvini, S. Forte, P. Groth Merrild, J. Rojo and L. Rottoli, Intrinsic charm in a matched general-mass scheme, Phys. Lett. B 754, 49 (2016).

[20] T. Boettcher, P. Ilten and M. Williams, A direct probe of the intrinsic charm content of the proton, arXiv:1512.06666 [hep-ph].

[21] B. A. Kniehl, G. Kramer, I. Schienbein and H. Spiesberger, Open charm hadroproduction and the charm content of the proton, Phys. Rev. D 79, 094009 (2009).

[22] V. P. Gonalves, Theoretical aspects of heavy-flavour production at ultra-high cosmic ray energies, EPJ Web Conf. 99, 07001 (2015).

[23] A. Abdel-Rehim et al. [ETM Collaboration], Direct Evaluation of the Quark Content of the Nucleon from Lattice QCD at the Physical Point, arXiv:1601.01624 [hep-lat].

[24] M. Gong et al. [XQCD Collaboration], Strangeness and charmness content of the nucleon from overlap fermions on 2+1-flavor domain-wall fermion configurations, Phys. Rev. D 88, 014503 (2013).

[25] W. Freeman and D. Toussaint [MILC Collaboration], Intrinsic strangeness and charm of the 
nucleon using improved staggered fermions, Phys. Rev. D 88, 054503 (2013).

[26] C. S. An and B. Saghai, Sea flavor content of octet baryons and intrinsic five-quark Fock states, Phys. Rev. C 85, 055203 (2012).

[27] C. S. An and B. Saghai, Pion- and strangeness-baryon $\sigma$ terms in the extended chiral constituent quark model, Phys. Rev. D 92, 014002 (2015).

[28] A. Le Yaouanc et al., Naive quark pair creation model of strong interaction vertices, Phys. Rev. D 8, 2223 (1973) ; A. Le Yaouanc et al., Naive quark pair creation model and baryon decays, Phys. Rev. D 9, 1415 (1974) ; R. Kokoski and N. Isgur, Meson Decays by Flux Tube Breaking, Phys. Rev. D 35, 907 (1987).

[29] L. Y. Glozman and D. O. Riska, The Spectrum of the nucleons and the strange hyperons and chiral dynamics, Phys. Rept. 268, 263 (1996).

[30] L. Y. Glozman and D. O. Riska, The Charm and bottom hyperons and chiral dynamics, Nucl. Phys. A 603, 326 (1996); Erratum: [Nucl. Phys. A 620, 510 (1997)].

[31] S. G. Yuan, C. S. An, K. W. Wei, B. S. Zou and H. S. Xu, Spectrum of low-lying $s^{3} Q \bar{Q}$ configurations with negative parity, Phys. Rev. C 87, 025205 (2013).

[32] C. S. An and B. Saghai, Strangeness magnetic form factor of the proton in the extended chiral quark model, Phys. Rev. C 88, 025206 (2013).

[33] K. A. Olive et al. [Particle Data Group Collaboration], Review of Particle Physics, Chin. Phys. C 38, 090001 (2014).

[34] R. S. Towell et al. [NuSea Collaboration], Improved measurement of the $\bar{d}-\bar{u}$ asymmetry in the nucleon sea, Phys. Rev. D 64, 052002 (2001).

[35] B. Borasoy and U. -G. Meissner, Chiral expansion of baryon masses and sigma terms, Annals Phys. 254, 192 (1997).

[36] W. C. Chang and J. C. Peng, Flavor Asymmetry of the Nucleon Sea and the Five-Quark Components of the Nucleons, Phys. Rev. Lett. 106, 252002 (2011); W. C. Chang and J. C. Peng, Extraction of Various Five-Quark Components of the Nucleons, Phys. Lett. B 704, 197 (2011).

[37] L. Shao, Y. -J. Zhang and B. -Q. Ma, Sea quark contents of octet baryons, Phys. Lett. B 686, 136 (2010).

[38] A. Semke and M. F. M. Lutz, Strangeness in the baryon ground states, Phys. Lett. B 717, 242 (2012); M. F. M. Lutz et al., Finite volume effects in the chiral extrapolation of baryon masses, Phys. Rev. D 90, 054505 (2014). 
[39] X. L. Ren et al., Virtual decuplet effects on octet baryon masses in covariant baryon chiral perturbation theory, Phys. Rev. D 87, 074001 (2013); X. L. Ren et al., Scalar strangeness content of the nucleon and baryon sigma terms, Phys. Rev. D 91, 051502 (2015).

[40] S. Durr et al., Sigma term and strangeness content of octet baryons, Phys. Rev. D 85, 014509 (2012); R. Horsley et al. (QCDSF-UKQCD Collaboration), Hyperon sigma terms for $2+1$ quark flavours, Phys. Rev. D 85, 034506 (2012); G. S. Bali et al. (QCDSF Collaboration), The strange and light quark contributions to the nucleon mass from Lattice QCD, Phys. Rev. D 85, 054502 (2012); C. Alexandrou et al., The quark contents of the nucleon and their implication for dark matter search, PoS LATTICE 2013, 295 (2014).

[41] J. J. Aubert et al. [European Muon Collaboration], An Experimental Limit on the Intrinsic Charm Component of the Nucleon, Phys. Lett. B 110, 73 (1982); J. J. Aubert et al. [European Muon Collaboration], Production of charmed particles in $250-\mathrm{GeV} \mu^{+}$- iron interactions, Nucl. Phys. B 213, 31 (1983).

[42] C. Adloff et al. [H1 Collaboration], Inclusive D0 and D*+- production in deep inelastic e p scattering at HERA, Z. Phys. C 72, 593 (1996).

[43] J. Breitweg et al. [ZEUS Collaboration], D* production in deep inelastic scattering at HERA, Phys. Lett. B 407, 402 (1997).

[44] S. J. Brodsky and S. Gardner, Comment on "New Limits on Intrinsic Charm in the Nucleon from Global Analysis of Parton Distributions", Phys. Rev. Lett. 116, 019101 (2016).

[45] P. Jimenez-Delgado, T. J. Hobbs, J. T. Londergan and W. Melnitchouk, Reply to Comment on "New limits on intrinsic charm in the nucleon from global analysis of parton distributions", Phys. Rev. Lett. 116, 019102 (2016).

[46] S. J. Brodsky, A. S. Goldhaber, B. Z. Kopeliovich and I. Schmidt, Higgs Hadroproduction at Large Feynman x, Nucl. Phys. B 807, 334 (2009).

[47] S. G. Yuan, K. W. Wei, J. He, H. S. Xu and B. S. Zou, Study of $q q q c \bar{c}$ five quark system with three kinds of quark-quark hyperfine interaction, Eur. Phys. J. A 48, 61 (2012); B. S. Zou, Hadron spectroscopy from strangeness to charm and beauty, Nucl. Phys. A 914, 454 (2013).

[48] R. D. Ball, M. Bonvini and L. Rottoli, Charm in Deep-Inelastic Scattering, JHEP 1511, 122 (2015).

[49] J. Butterworth et al., PDF4LHC recommendations for LHC Run II, J. Phys. G 43, 023001 (2016). 